\title{
GENERALIZED ANALYTIC FUNCTIONS
}

BY

\author{
RICHARD ARENS AND I. M. SINGER
}

1. Introduction. The theory of functions holomorphic in the unit disc

$$
f(z)=a_{0}+a_{1} z+a_{2} z^{2}+\cdots \quad(|z|<1)
$$

can be regarded as emanating from the study of such trigonometrical series

$$
1.2 \sum \sum a_{n} e^{i n t}
$$

where $a_{n}=0$ for $n<0$. Abstract harmonic analysis treats the generalization of 1.2 where the unit circle is replaced by any (abelian) locally compact group $\Gamma$ while the set of indices $(n)$ is taken as the character group, $G$, of $\Gamma$.

Just as 1.1 is obtained from 1.2 by requiring the $\left\{\cdots, a_{-2}, a_{-1}, a_{0}, a_{1}\right.$, $\left.a_{2}, \cdots\right\}$ to be nonzero only on the subset $0,1,2, \cdots$, so we study here the special properties of algebras of functions (the operations being addition and convolution on $G$ and addition and multiplication on $\Gamma$ ) whose support in $G$ lies always in a fixed semi-group $G_{+}$of $G$ (corresponding to the class of indices $0,1,2, \cdots$ in the classical case). The general idea is to note the form in which classical objects reappear.

The disc $|z| \leqq 1$ becomes the space $\Delta$ of maximal ideals of the function algebra; the group $\Gamma$ becomes the boundary of the disc, and all functions take their maximum modulus on $\Gamma$.

For interior points $\zeta$ of the disc, there is a measure on $\Gamma$ such that the value at $\zeta$ is given by an integral of the boundary values. This "Poisson integral" is studied in detail, and it is shown that one of these "generalized holomorphic" functions $f$ cannot vanish on an open set of the boundary which has positive measure with respect to all of these harmonic measures. Moreover, when $G$ has sufficiently many continuous "characters" (with values in the unit disc), and $f$ vanishes on a nonvoid open set, then $f=0$. In particular, the algebra becomes an integral domain. We can thus make arbitrarily huge (semi-simple) Banach algebras which are integral domains.

We wish to cite some work related to ours done by Goldman [VI] and by Mackey [VII]. Goldman states 3.1 when $G$ is a totally ordered discrete group. Moreover, our 4.6 was motivated by a discussion with him concerning possible generalizations of the classical case of the disc. Mackey also states 3.1 and has a complicated Cauchy integral formula. His point of view is much

Received by the editors August 20, 1955. 
different from ours; briefly put, he considers all the orderings on $G$ at the same time, whereas we assume a given $G_{+}$as intrinsic part of the structure of $G$.

In later papers we shall present results on the automorphisms of the algebras considered here, their ideal theory, and generalizations of other classical theorems in complex variables.

2. The algebras to be studied. Let $G$ be a locally compact abelian group, and let $G_{+}$be a subset of $G$ such that

2.1. $G_{+}$is a closed subset of $G$;

2.2. the interior of $G_{+}$is dense on $G_{+}$and also generates $G$;

2.3. $x, y$ in $G_{+}$implies $x y$ in $G_{+}$.

Let $A_{1}$ be the algebra (under convolution, using the left-invariant Haar measure) of those summable functions which vanish outside $G_{+}$. Then $A_{1}$ is clearly a closed subalgebra of $L_{1}(G, C)$, and is a Banach algebra with the norm

$$
\|f\|_{1}=\int_{G}|f(x)| d x .
$$

As is well known (cf. III), elements of $L_{1}$ determine operators in $L_{2}(G, C)$, wherein $f$ gives rise to $T_{f}, T_{f}(g)=f * g$. The bound of the operator $T_{f}$ we will denote by $|f|$. Then $|f| \leqq\|f\|_{1}$. The completed operator algebra will be denoted by $A_{0}$. Both algebras are invariant under translation by elements of $G_{+}$, that is, if $f_{a}(x)=f(a x), f^{a}(x)=f\left(x a^{-1}\right)$, then $f$ in $A_{1}$ gives $f_{a}$ in $A_{1}$ if $a^{-1}$ is in $G_{+}$and gives $f^{a}$ in $A_{1}$ if $a$ is in $G_{+}$.

These algebras are subalgebras of well-known algebras, and are both representable as algebras of continuous functions, vanishing at infinity, on the character group $\Gamma$ of $G$. It is not easy, in general, when looking at a particular $\phi$ in $\mathcal{C}_{0}(\Gamma, C)$ (= continuous functions on $\Gamma$ vanishing at infinity, complex values) to tell whether it is a representing function-particularly not for the case of $A_{1}$. When $G$ is the group of integers and $G_{+}$is the set of nonnegative integers, then the representing functions are those defined on the unit circle and (extendable to) the interior so as to have absolutely convergent Taylor series [III, p. 81]. The situation for $A_{0}$ is always simpler. In the special case mentioned, the functions one gets are just the continuous functions on the unit circle which can be extended to the interior so as to be analytic there, or what is the same thing, whose Fourier coefficients

$$
\frac{1}{2 \pi} \int_{0}^{2 \pi} \phi\left(e^{i t}\right) e^{-i n t} d t
$$

vanish for $n=-1,-2, \cdots$.

In general, the norm in $A_{0}$ is exactly the "sup" norm of the Fourier transform 


$$
\widehat{f}(\alpha)=\int_{G} f(x)(\alpha, x) d x
$$

for those operators $T_{f}$ which arise from $f$ in $A_{1}$. This results from a consideration of the Plancherel theorem [III, p. 145]. Loosely speaking one obtains, even after completion, those functions in $\mathcal{C}_{0}(\Gamma, C)$ (defined above) whose (inverse) Fourier transforms are supported on $G_{+}$. Rather than make this precise, we content ourselves with the following which is completely adequate when $G$ is discrete.

2.6. Theorem. Any function $\phi$ in $\mathcal{C}_{0}(\Gamma, C)$ which is summable and uniformly continuous corresponds to an operator $T$ in $A_{0}$ (under the representation defined where possible by 2.5$)$, provided for all $x$ not in $G_{+}$:

$$
\int \phi(\alpha) \overline{(\alpha, x)} d x=0 .
$$

Proof. Obtain a continuous $\psi$ with compact support such that 2.7

$$
\|\psi * \phi-\phi\|_{\infty}<\epsilon .
$$

Then $\psi=\widehat{g}$ where $g$ is in $L_{2}(G)$. Moreover, $g f$ is in $L_{1}\left(G_{+}\right)$and the bound of the operator $T_{g f}-T$ is given by 2.7 .

Finally, because of 2.2, we can also say this:

2.8. TheOREM. The functions representing $A_{1}$ (and a fortiori, $A_{0}$ ) separate $\Gamma$.

Because $\Gamma$ is contained in char $G_{+}, 2.8$ also follows from 4.1, below.

3. Characters of $G_{+}$. When $G$ is abelian we can call a character of $G_{+}$any continuous complex valued function $\xi$ on $G_{+}$such that $\xi(x y)=\xi(x) \xi(y)$, $|\xi(x)| \leqq 1$, which is not (identically) zero. This generalizes the ordinary concept of character group (when $G_{+}=G$ ); and many notions of the theory of character groups may be carried over. In particular, the class of these, to be called $\Delta$, is locally compact, and a Hausdorff space, with the topology of uniform convergence on compact sets.

Because of condition 2.2, characters of $G_{+}$have a polar decomposition exemplified in the classical case by writing $z=r e^{i \theta}$.

3.1. Theorem. Let $G$ be an abelian locally compact group, and let 2.1, 2.2, 2.3 hold. Then any character $\zeta$ of $G_{+}$can be written as a product

$$
\zeta=\rho \alpha(=\alpha \rho)
$$

where $\alpha$ is a character of the whole group $G$, while $\rho$ is a uniquely-determined nonnegative character of $G_{+}$.

Proof. The "modulus" $\rho$ can be at once obtained as $\rho(x)=|\zeta(x)|$. We have now to extend 
from that part of $G$ on which it is uniquely determined, to a character of $G$. If $\zeta \neq 0$, then $\zeta(x) \neq 0$ on some open part (of $G_{+}$) which generates an open subgroup $G_{0}$ (of $G$ ) on which 3.3 is uniquely definable yielding a continuous character of $G_{0}$. This can be extended to all of $G$ [IV, p. 142] (the extension is not necessarily unique). In any case, the $\rho$ is unique.

4. Complex homomorphisms of $A_{0}$ and $A_{1}$. Let $\operatorname{Hom}(A, C)$ or, more briefly, Hom (A) be the space of continuous homomorphisms of a normed algebra $A$ on the complex numbers $C$. One of the central results of general harmonic analysis [cf. III, p. 136] is that for a locally compact abelian group $G$, if $A=L_{1}(G)$ then Hom (A) corresponds in a natural one-to-one way to $\Gamma$. In this section, we show that the same type of correspondence holds between Hom $\left(A_{1}\right)$ (and also Hom $\left(A_{0}\right)$ ) and $\Delta$. This new result generalizes the known result about $L_{1}(G)$, since one may take $G_{+}=G$. On the other hand, the generalization is so easy (although independent) that we can afford to give a brief exposition.

4.1. Theorem. There is a homeomorphism between Hom $\left(A_{1}\right)$ (and Hom $\left(A_{0}\right)$ ) and $\Delta$, of a form given below.

Proof. A continuous homomorphism of $A_{0}$ immediately defines one of $A_{1}$, so we may begin by taking a continuous homomorphism $\xi$ of $A_{1}$. Select an $f$ in $A_{1}$ such that $\xi(f) \neq 0$. Define

$$
\zeta(x)=\xi\left(f^{x}\right) / \xi(f)
$$

$\left(x\right.$ in $\left.G_{+}\right)$.

(For $f^{x}$ see $\S 2$.) This is a continuous character of $G_{+}$and does not depend on the $f$ chosen.

Now begin with a $\zeta$ from $\Delta$. We define

$$
\xi(f)=\int_{G} f(x) \zeta(x) d x
$$

for $f$ in $A_{1}$. This obviously defines an element of Hom $\left(A_{1}\right)$. It is very easy to see that the relation of $\zeta$ to $\xi$ is given also by 4.2 .

The dual question, whether the definition 4.2 implies the relation 4.3 , is treated as follows. Let 4.2 hold. Then

$$
\int \zeta(x) f(x) d x \cdot \xi(f)=\int \xi\left(f^{x} f(x)\right) d x .
$$

Making use of the fact that $\xi(h)=\int_{G} h(t) m(t) d t$, for some bounded measurable $m$, we can interchange the order of integration on the right, and obtain

$$
\int f(x) \zeta(x) d x \cdot \xi(f)=\xi(f * f)
$$

which gives 4.3 .

Every continuous homomorphism $\xi$ of $A_{1}$ is bounded in the $A_{0}$-norm, since 
as the Plancherel theorem shows.

There remains the question of whether the locally compact spaces $\Delta$ and Hom $\left(A_{1}\right)$, Hom $\left(A_{0}\right)$ are homeomorphic under these mappings. Considering $A_{1}$, first, we state that the proof of [III, 34C] can be immediately adapted (because the compact set $C$ can be chosen inside $G_{+}$, and the integral 4.3 can be used). Finally, Hom $\left(A_{1}\right)$ and Hom $\left(A_{0}\right)$ are already in one-one correspondence, and a subbase for the weak topology induced by $A_{0}$ can be chosen using only (the densely situated) elements coming from $A_{1}$. This completes the proof of 4.1 .

We shall henceforth always consider $\Delta$ as the space Hom $\left(A_{1}\right)$ or Hom $\left(A_{0}\right)$ itself, and may even call the points thereof maximal ideals of these algebras.

Different characters of $G$ will be distinct even on $G_{+}$. Moreover, the topology is also the same (because of 2.2). The subset of $\Delta$ corresponding to $\Gamma$ will be denoted also by $\Gamma$.

The analogy to analytic functions begins to take a more concrete form with the following statement.

4.6. Theorem. For either algebra, $\Gamma$ is the Silov boundary in (or, of) the space of maximal ideals $\Delta$. In other words, for each element $f$ (of $A_{1}$, for example) the maximum modulus of the representing function

\subsection{1}

is taken on the set $\Gamma$; and $\Gamma$ is the least closed set in $\Delta$ having this property (uniformly for all ring elements).

Proof. The maximum modulus is in any case given by (for an element $f$ in $\left.A_{1}\right)$

$$
\lim \left\|f^{(n)}\right\|_{1}^{1 / n}=|f|
$$

and this is certainly taken on $\Gamma$, since it is the space of maximal ideals of $L_{1}(G)$ as a whole. Therefore $\Gamma$ contains the Silov boundary $F$, which is not void (see [III, 24E]). Now the class of representing functions is surely invariant under translation in $\Gamma$ since this means merely multiplication by characters in $G$ and does not move the support off $G_{+}$. Thus $F$ itself is translation invariant, and hence must be all of $\Gamma$.

The reader may find it convenient to associate " $\Delta$ " with "disc" and " $\Gamma$ " with Silov's word for "boundary."

The situation presented in 4.6 fulfills the condition of a general result [I, 7.1], showing that Poisson-integral-like representations are possible.

4.7. Theorem. For each point $\zeta$ of the disc $\Delta$ there is a measure $m_{\zeta}$ (in this paper, that shall always mean a regular Baire measure) defined on the boundary $\Gamma$ such that for each representing function of an element of $A_{0}$ (and thus, $a$ fortiori, of $\left.A_{1}\right), \phi$, 


$$
\int_{\Gamma} \phi(\alpha) m_{\zeta}(d \alpha)=\phi(\zeta)
$$

Moreover, for any such measure satisfying 4.71 (we shall say $m_{\zeta}$ represents $\zeta$ ) and for each $x$ in $G_{+}$there holds

$$
\int_{\Gamma}(\alpha, x) m_{\zeta}(d \alpha)=\zeta(x) .
$$

Finally, if $m_{\zeta}, m_{\eta}$ represent $\zeta, \eta$ (respectively) then the convolution (produit de composition [V, p. 47]) of these measures represents $\zeta \eta$.

Proof. There remains merely to derive 4.72 as well as the last assertion from 4.71. 4.72 results from using an "approximate identity" [III] translated to an interior point of $G_{+}$, followed by a limit. The remainder is a simple formal matter. It says in particular that if $\eta$ belongs to $\Gamma$ itself (so that the passage from $\zeta$ to $\zeta \eta$ is a "rotation") then the measure for $m_{\zeta}$ can be rotated to provide one for $\zeta \eta$.

4.8. Corollary. If $G_{+} \cup G_{+}^{-1}=G$ then the measure, for each $\zeta$, is unique.

Proof. Although Radon-measures (complex valued) can be found to represent $\zeta, 4.7$ mentions only real ones. For these, taking conjugates in 4.72 yields

$$
\int_{\Gamma}(\alpha, x) m_{\zeta}(d \alpha)=\zeta(x)
$$

for $x^{-1}$ in $G_{+}$, and thus for all $x$. Now a measure is determined by its FourierStieltjes coefficients (left side of 4.81 ), which are by 4.81 the same for all measures representing $\zeta$.

An example of nonunique measures can be easily given [I, p. 740].

We have not seriously attempted to survey the (convex closed) set of measures representing a given $\zeta$. In the next section we provide an independent proof of the existence of one particularly attractive measure (for each $\zeta$ ), to be called the harmonic measure, at this stage merely on its aesthetic merit.

5. The harmonic measures. Let $\rho$ be a point of the "positive axis" in the disc, that is, a character of $G_{+}$which is real and non-negative. We wish to construct a particularly simple measure representing $\rho$.

There is an open subgroup $G_{\rho}$ of $G$ generated by those points of $G_{+}$at which $\rho$ is positive. A unique continuous homomorphism

\section{1}

of $G_{\rho}$ into the group of positive real numbers exists, which is an extension of $\rho$ to $G_{\rho}$.

By an adaptation of Pontrjagin's argument [IV, p. 142, Lemma] we can extend this to a continuous homomorphism 
5.11

of the whole group $G$ into the positive reals. (This extension is not always unique, but the result will be independent of the manner of it.)

Then, for each real number $v$, we can define a character of $G$

$$
k(v)=r^{i v}
$$$$
(-\infty<v<\infty)
$$

where $(k(v), x)=e^{i v \log r(x)}, x \in G$.

Now let $\Gamma_{\rho}$ be the group of characters of $G$ which are 1 on $G_{\rho}$. Since $G_{\rho}$ is open, $\Gamma_{\rho}$ is compact (being isomorphic to the character group of $G / G_{\rho}$ ). Moreover, if $G_{\rho}=G, \Gamma_{\rho}$ reduces to the identity.

Recall the function

$$
c(v)=\left[\pi\left(1+v^{2}\right)\right]^{-1}
$$

(known as Cauchy's probability density [II, 19.2.1]). It has the property that its Fourier transform

$$
\hat{c}(x)=e^{-|x|}
$$

We are now ready to define an integral $I_{\rho}$ representing $\rho$. For $\phi$ any complex-valued, bounded Baire function on $\Gamma$, let

$$
I_{\rho}(\phi)=\int_{-\infty}^{\infty} \int_{\Gamma_{\rho}} \phi\left(\gamma r^{i v}\right) d \gamma c(v) d v .
$$

The first integration is with respect to the normalized Haar measure of $\Gamma_{\rho}$, and not with respect to the Haar measure of $\Gamma$ (which wouldn't make sense most of the time anyway. Sometimes $\Gamma_{\rho}=\Gamma$, however.)

Formula 5.3 clearly defines an integral on $\Gamma$. Moreover,

$$
0 \leqq \phi \leqq 1 \text { implies } 0 \leqq I_{\rho}(\phi) \leqq 1 .
$$

Our primary concern is whether, for $f$ in $A_{0}$ or $A_{1}$, we have

$$
I_{\rho}(\widehat{f})=\rho(f) .
$$

Since (at least for $f$ in $A_{1}$ ) we have 2.5 , it will clearly suffice to prove that 5.33

$$
I_{\rho}(x)=\rho(x)
$$

where $x$ on the left is the bounded continuous function defined, of course, by $x(\alpha)=(\alpha, x)$. Then 5.32 will follow by inversion of the order of some integrations for $f$ in $A_{1}$, and for $A_{0}$, by uniform approximation and use of 5.31.

Now (for $x \in G_{+}$)

5.34

$$
I_{\rho}(x)=\iint\left(\gamma r^{i v}, x\right) d \gamma c(v) d v
$$

$$
=\int_{\Gamma_{\rho}}(\gamma, x) d \gamma \cdot \int e^{i v \log r(x)} c(v) d v
$$


The first factor is 0 for $x \notin G_{\rho}$, and so is $I_{\rho}(x)$. When $x \in G_{\rho}$, then $(\gamma, x)=1$ for all $\gamma$ in $\Gamma_{\rho}$, so the first integral is 1 . The second (by 5.21) is

$$
\min \left(r(x), r(x)^{-1}\right)
$$

and for $x$ in $G_{+} \cap G_{\rho}$, this reduces to $\rho(x)$ as desired, since $|\rho(x)| \leqq 1$.

We shall also compute the Fourier-Stieltjes transform of (in classical terminology, the measure $\mu_{\rho}$ corresponding to) $I_{\rho}$. We use the formula 5.34, and obtain the result

$$
I_{\rho}(x)= \begin{cases}0 & \left(x \notin G_{\rho}\right) \\ \min \left(r_{\rho}(x), r_{\rho}(x)^{-1}\right) & \left(x \in G_{\rho}\right) .\end{cases}
$$

Here the values of $r_{\rho}$ are uniquely determined by $\rho$, since we are on $G_{\rho}$. Thus $I_{\rho}$ and $\mu_{\rho}$ are independent of the mode of construction of $r$.

For a point $\zeta \in \Delta$ which is not real and non-negative, we perform first the polar decomposition

$$
\zeta=\rho \alpha
$$$$
(\rho=|\zeta|, \alpha \in \Gamma)
$$

and define

$$
I_{\zeta}(\phi)=I_{\rho}\left(\phi^{\alpha}\right)
$$

where $\phi^{\alpha}(\beta)=\phi(\beta \alpha)$ for $\phi$ as before (5.3).

We formulate our findings in the following way.

5.5. ThEOREM. Let $\zeta$ be any point of $\Delta$. Then there is an integral $I_{\zeta}$ on $\mathrm{I}$ which represents $\zeta$ (in the sense of 4.7), having these properties:

For a bounded Baire function on $\Gamma$,

$$
I_{\zeta}(\phi)=\int_{-\infty}^{\infty} \int_{\Gamma_{\zeta}} \phi\left(\gamma \alpha r^{i v}\right) d \gamma c(v) d v
$$

where $\Gamma_{\zeta}$ is the (compact) subgroup of $\Gamma$ of those characters which are 1 on the subgroup $G_{\zeta}$ generated in $G$ by those $x$ in $G_{+}$on which $\zeta(x) \neq 0$, and " $d \gamma$ " refers to the normalized Haar measure in $\Gamma_{p}$; and $\alpha$ is selected so that 5.4 holds, while $r$ is any extension (5.11) to $G$ of the unique continuous multiplicative homomorphism defined by $\rho$ on $G_{\zeta}$, to all of $G$; and $c(v)$ is Cauchy's distribution 5.2.

The Fourier-Stieltjes transform of the measure $\mu_{\zeta}$ associated with 5.51 is as follows:

$$
\int_{\Gamma}(\beta, x) \mu_{\zeta}(d \beta)= \begin{cases}0, & x \notin G_{\zeta}, \\ (\beta, x) \min \left(r(x), r(x)^{-1}\right), & x \in G_{\zeta},\end{cases}
$$

(and it should be borne in mind that $r(x)$ is uniquely determined by $\zeta$ for $x$ in $G_{\zeta}$ ).

It is formally interesting that the combination $\alpha r^{i v}$ is an extension of $\zeta|\zeta|^{i v-1}$. 
The measure $\mu_{\zeta}$ we might call the harmonic measure for $\zeta$. This is only a slight abuse of classical terminology. The integral itself is of course to be called the Poisson integral (with parameter $\zeta$ ).

Let us consider briefly the carrier of an harmonic measure. We first look at it from a measure-theory position. The measure $\mu_{\rho}$ is evidently the "product of composition" [V, p. 47] of the Haar measure of $\Gamma_{\rho}$ (regarded now as a measure in $\Gamma$, usually singular with respect to the proper Haar measure of $\Gamma$ ) and the measure obtained by placing Cauchy's distribution on the oneparameter subgroup 5.12. Each one of these two subgroups may degenerate down to the identity. For example, if $\rho(x)$ is never 0 , then $\Gamma_{\rho}=\{1\}$, while if $\rho(x)$ is identically 1 on $G_{\rho}$ (and of course 0 outside), then $r$ (see 5.11) can be chosen to be 1 . Thereupon the group 5.12 degenerates, and $\mu_{\rho}$ becomes the Haar measure of $\Gamma_{\rho}$. This case occurs precisely when $\rho=\rho \rho$, and is not limited to the case $\rho=0$ or 1 in general. (Of course it is so limited when $G$ is archimedean linear ordered and $G_{+}$is the set of elements not less than the neutral element.)

In the topological sense, the carrier of a Baire measure is the complement of the union of all open sets of measure 0 . For the harmonic measures, this is not usually as small as the subgroup of 5.12 (even when $\Gamma_{\rho}=\{1\}$ ) for this need not be closed, nor is it usually as big as $\Gamma$, as the previous paragraph points out. It is, however, actually a (closed) subgroup, which we now describe.

Return to a $\rho$ as at the start of this section, and consider the subgroup $G^{\rho}$ of $G_{\rho}$ consisting of those $x$ in $G_{\rho}$ at which $r(x)=1$ (see 5.11). This is closed (but not generally open). Let $\Gamma^{\rho}$ be the class of those $\alpha$ in $\Gamma$ which are 1 on $G^{\rho}$. This, too, is closed (but not always compact).

5.6. Lemma. Let $H$ be the class of all $\alpha$ in $\Gamma$ for each of which a real number $v$ can be found such that $(\alpha, x)=r(x)^{\text {iv }}$ for all $x$ in $G_{\rho}$. Then $H$ is a dense subgroup of $\Gamma^{p}$.

Proof. Any $\alpha$ of this form is certainly 1 on $G^{\rho}$, whence $H$ is included in $\Gamma^{\rho}$. Suppose for a moment that it were not dense.

Then there exists a continuous character of $\Gamma$ which is 1 on $H$, and not identically 1 on $\Gamma^{\rho}$. (We apply twice Pontrjagin's [loc. cit.] extension theorem for closed subgroups.) This character is an element $x$ of $G$. If $x \notin G_{\rho}$, we could make a character $\alpha$ that is 1 on $G_{\rho}$ and not 1 on $x$. This $\alpha$ belongs to $H(v=0)$, so we reject the possibility, and conclude $x \in G_{\rho}$. For every real $v, r(y)^{i v}$ always defines an element of $H$. Hence $r(x)^{i v}=1$ (for all $v$ ), which implies $r(x)=1$, or $x \in G^{\rho}$. But then $x$ defines the unit character on $\Gamma^{\rho}$. This contradiction shows that $H$ is dense in $\Gamma^{p}$.

Thus 5.6 is proved.

Now suppose $\phi \geqq 0$ and $\phi(\alpha)>0$ for some $\alpha$ in $\Gamma^{p}$. Then for at least for a 
nonvoid, open set of pairs $(v, \gamma)\left(\gamma \in \Gamma_{\rho}\right)$ we have $\phi\left(\gamma r^{i v}\right)>0$, by 5.6. Hence $I_{\rho}(\phi)$ is surely positive. This enables us to assert the following.

5.7. TheOREM. The carrier of the measure defining the integral $I_{5}(\zeta=\rho \alpha$ in its polar decomposition) is the coset $\alpha \Gamma^{\rho}$ of the closed subgroup $\Gamma^{\rho}$ of $\Gamma$.

5.71. Corollary. Let $G$ be a linearly ordered group. Let $\zeta \in \Delta$ be such that $|\zeta(x)| \neq 1$ except for $x=1$. Then every open nonvoid set in $\Gamma$ has positive measure with respect to the measure defining $I_{5}$.

Proof. $G^{\zeta}$ reduces to the identity in this case, and so $\Gamma^{\zeta}=\Gamma$. Hence the carrier, being just a coset, is $\Gamma$.

In a general context, it was established [I, 7.1] that there always exist representing measures $m$ such that, for any representing function $\phi$ for the ring $A_{0}$ (and hence $a$ fortiori for $\phi=\widehat{f}, f \in A_{1}$ )

$$
\log \left|\int_{\Gamma} \phi(\alpha) m(d \alpha)\right| \leqq \int_{\Gamma} \log |\phi(\alpha)| m(d \alpha)
$$

or

$$
\log \left|I_{\zeta}(\phi)\right| \leqq I_{\zeta}(\log |\phi|) .
$$

Both sides may have the value $-\infty$. The left side is of course log $|\phi(\zeta)|$ if the measure $m$ is supposed to represent $\zeta$.

We are about to assert that this property 5.72 is enjoyed by our harmonic measures. There would be no need for anything more than a reference to [I, 7.1] if $G_{+} \cup G_{+}^{-1}$ were all of $G$, for then by 5.52 there could be no other measure than $\mu_{\zeta}$ representing $\zeta$ and so $\mu_{\zeta}$ would have the property 5.72 predicated for at least one such measure [I, 7.1]. The general case will be reduced to this special situation.

5.8. Theorem. For each $\zeta$, the measure $\mu_{\zeta}$ satisfies 5.72 and 5.73 whenever $\phi$ is a function representing on $\Delta$ any element of $A_{0}$ or of $A_{1}$.

Proof. It will suffice to consider $\zeta$ of the form of $\rho$. Let an $r$ (see 5.11) be constructed. Let us enlarge $G_{+}$to the set $G_{1}$ generated by $G_{+}$and those places where $r(x) \leqq 1$. Then $G$ satisfies the conditions 2.1-2.3 and gives rise to a new $\left(A_{0}\right)_{1}$ containing the old $A_{0}$. The new $\Delta_{1}$ is contained in the old $\Delta$, but the Silov boundary is still $\Gamma$.

Now define $\sigma(x)=0$ for $x \in G_{1}$ but $x \notin G_{\rho}$, and $\sigma(x)=r(x)$ for $x \in G_{\rho}$ and $r(x) \leqq 1$. Then $\sigma \in \Delta_{1}$, and $\sigma$, confined to $A_{0}$, is the original $\rho$. The subgroups $G_{\sigma}, \Gamma_{\sigma}$ are the same as for $\rho$, and the same $r$ can be used to define $I_{\sigma}$. But $G_{1} \cup G_{1}^{-1}=G$ (with even some overlap, but this was never excluded) so that there is only one $I_{\sigma}$ possible (by 5.52) and so (as remarked earlier), by [I, 7.1], 5.72 and 5.73 must hold for a function representing an element of $\left(A_{0}\right)_{1}$, and $\mu_{\sigma}$. But by 5.3 or even $5.36, I_{\sigma}=I_{\rho}$, and on the other hand, $A_{0}$ is a part of $\left(A_{0}\right)_{1}$. Thus 5.8 is proved. 
6. Homomorphisms. Suppose we have two locally compact abelian groups $G, H$ with $G_{+}, H_{+}$subsets satisfying $2.1-2.3$; and suppose there is a homomorphism

$$
\alpha: G \rightarrow H
$$

preserving the relevant structure, that is

$$
\alpha\left(G_{+}\right) \subset H_{+} .
$$

Then, of course, we obtain dual homomorphisms of the discs

\subsection{1}

$$
\alpha^{*}: \Delta_{H} \rightarrow \Delta_{G}
$$

and of the character groups

$$
\alpha^{*}: \Gamma_{H} \rightarrow \Gamma_{G} .
$$

Let $\sigma \in \Delta_{H}$. We intend $\alpha^{*}(\sigma)(x)=\sigma(\alpha(x))$ for $x$ in $G$, by 6.11 . Let us construct the Poisson integrals for $\sigma$ and $\alpha^{*}(\sigma)=\sigma \circ \alpha$. Now let $\phi$ be a bounded Baire function on $\Gamma_{a}$. Then $\psi$, defined by $\phi \circ \alpha^{*}$, is a function of the same sort on $\Gamma_{H}$. The natural thing would be

$$
I_{\sigma}\left(\phi \circ \alpha^{*}\right)=I_{\sigma \circ \alpha}(\phi)
$$

and this would be immediate if there were only one way, in each case, of extending the Poisson integrals from the class of representative functions (but this is not generally true, as we have pointed out before). 6.2 can be proved, nevertheless.

\subsection{Theorem. In the circumstances above, 6.2 is true.}

We provide only that part of the proof which is not automatic, in that it involves an arbitrary extension. We prove

$$
6.31
$$

$$
\alpha^{*}\left(\Gamma_{\sigma}\right)=\Gamma_{\sigma \circ \alpha} .
$$

We may presume $\sigma$ here of the form $0 \leqq \sigma$. It is rather clear that " $\subset$ " holds in 6.31. Approaching the converse, we select $\beta$ from the right-hand side of 6.31. Then $\beta=1$ on $G_{\sigma \circ \alpha}$ (by definition) and the latter quite evidently is $\alpha^{-1}\left(H_{\sigma}\right)$. Therefore $\beta$ defines a character of the discrete group $G / \alpha^{-1}\left(H_{\sigma}\right)$, which in turn is isomorphic (by $\alpha$, and an isomorphism theorem) to the discrete group $H / H_{\sigma}$. Thus we obtain a character of the latter which defines a character $B$ of $H$. Clearly $B$ belongs to $\Gamma_{\sigma}$ (more fully, $\left(\Gamma_{H}\right)_{\sigma}$ ) and also $\beta=B \circ \alpha=\alpha^{*}(B)$. This proves 6.31 .

The remainder of the proof consists merely of automatic manipulations with the two members of 6.2, each written in the form 5.3.

We insert here some comments on the possibility of a Poisson kernel for the integral.

In the classical case ( $G$ the integers, $G_{+}$the non-negative ones) the Poisson 
integral 5.43 has a kernel,

6.4

$$
\int_{\Gamma} \phi(\alpha) K_{\rho}(\alpha) d \alpha=I_{\rho}(\phi),
$$

integration being with respect to (usually, the normalized) Haar measure. In general, 6.4 is possible whenever $\mu_{\rho}$ is absolutely continuous with respect to Haar measure in $\Gamma$. For this to be possible, $G_{\rho}$ (see 5.42) must be $\sigma$-finite (since it carries the Fourier transform of the summable kernel $K_{\rho}$ ). However, we are not prepared to have a general discussion on this level.

We content ourselves with the following observation which deals with cases that can hardly be called bizarre.

6.5. Theorem. Let $G$ be discrete and have no elements of finite order (which is the same as saying that $\Gamma$ is compact and connected). Let $G_{\rho}=G$ for some $\rho$ in $\Delta$ (see 5.4). Then, unless $G$ is the integers and $\Gamma$ is the circle group, $\mu_{\rho}$ is singular with respect to the Haar measure of $\Gamma$.

Proof. Since $G_{\rho}=G$ there is no need to integrate over $\Gamma_{\rho}$, which reduces to one element. Hence the complement of the subgroup $\left\{r^{i v}\right\}$ (see 5.61) is of measure 0 . If this subgroup has infinitely many cosets, then it is of Haar measure 0 , and our measure is singular. In the contrary case, $\Gamma$ is exhausted by finitely many cosets. By a proof like that of the Baire density theorem, one of these must have an interior, so all are open. Because $\Gamma$ is connected, there can be only one, and the homomorphism sending $v$ into $r^{i v}$ must be open. It follows that it is not one to one, whence $\Gamma$ is the circle.

In the circumstances of 6.5 , there can clearly be no Poisson kernel unless we have the classical case. In particular, when $G$ is archimedean linearly ordered, and not the integers, there is a kernel only for that one $\rho$ in $\Delta$ (the center of the disc) whose measure is the Haar measure in $\Gamma$. This $\rho$ has $\rho(x)=0$ for $x>1$.

7. Mapping a half-plane into $\Delta$. Let $\rho$ be a non-negative element of $\Delta$. In terms of it, we can define (for $x$ in $G_{+}$)

$$
\rho^{0}(x)= \begin{cases}0 & \text { if } \rho(x)=0, \\ 1 & \text { if } \rho(x) \neq 0 .\end{cases}
$$

Then $\rho^{0} \in \Delta$, and $\rho=\rho \rho^{0}$. For real $v$ we have already considered $\rho^{i v}$, and we can also define $(u \geqq 0, v$ real $)$

$$
\rho^{u+i v}(x)= \begin{cases}\exp \{(\boldsymbol{u}+i v) \log \rho(x)\} & \text { if } \rho(x) \neq 0, \\ 0 & \text { if } \rho(x)=0 .\end{cases}
$$

This provides a continuous mapping of the half-plane $u \geqq 0$ into $\Delta$. To study its range (in $\Delta$ ) we recall the group $\Gamma^{\rho}$ (see 5.6). 


\subsection{TheOREM. For each $u \geqq 0$ the family}

7.21

$$
\left\{\rho^{u+i v} ; \text { vreal }\right\}
$$

lies densely in the subset $\rho^{u} \Gamma^{\rho}$ of $\Delta$.

Proof. The polar decomposition of $\rho^{u+i v}$ is of the form $\rho^{u} \alpha$. If $x \in G^{\rho}$ then $x=y z^{-1}$ where $y, z \in G_{\rho} \cap G_{+}$and $\rho(y)=\rho(z)$ whence $(\alpha, y)=(\alpha, z)$. Therefore $\alpha$ belongs to $\Gamma^{\rho}$, and $\rho^{u+i v}$ to $\rho^{u} \Gamma^{\rho}$.

Moreover, the $\alpha$ can be chosen to have the form $r^{i v}$ (see the proof of 5.6), hence the totality of these $\alpha$ is dense in $\Gamma^{\rho}$, by 5.6. And if the $\alpha$ is close to some $\beta$ (of $\Gamma$ ), then $\rho^{u+i v}$ is at least as close to $\rho^{u} \beta$, in the topology of $\Delta$. Hence the $\rho^{u+i v}$ are dense in $\rho^{u} \Gamma^{\rho}$, q.e.d.

When $G$ is archimedean-ordered then all but at most two $\rho$ 's satisfy the hypothesis in the following.

7.3. COROLlARY. If $\rho$ never assumes the same value more than once on $G_{+}$, then $\left\{\rho^{i v}\right\}$ lies densely in the boundary $\Gamma$.

For in this case $\Gamma^{\rho}=\Gamma$ while $\rho^{0}=1$.

We consider the effect of this mapping on the representing functions (4.71). The result is very simple.

7.4. TheOREM. Let $\phi$ be the representing function for an element of $A_{0}$. Then 7.41

$$
\phi\left(\rho^{u+i v}\right)
$$

is holomorphic for $u>0$, all $v$, and continuous and bounded for $u \geqq 0$, all $v$.

Proof. When $\phi$ represents an element $f$ of $A_{1}$, then 7.41 becomes

$$
\widehat{f}\left(\rho^{u+i v}\right)=\int_{G_{\rho}} f(x) \rho(x)^{u+i v} d x=f_{\rho}(u+i v) .
$$

This $f_{\rho}$ certainly has all the stated properties when $f$ has compact support. The function in the general case is the uniform limit of such $f_{\rho}$ as these, and hence shares the properties stated. Of course, the (operator) norm of the element in question provides an upper bound for the analytic function (and, in the circumstances of 7.3, the least upper bound).

In another paper we shall utilize the fact that when $G$ is discrete, these analytic functions are almost-periodic in the half-plane.

8. Boundary zeros. A function, continuous on the closed unit disc, holomorphic inside, and vanishing on an open subset of the boundary, vanishes identically. A simple proof of this well-known proposition is obtained by considering its Cauchy integral representation. Another is obtained by applying 5.72 to the Poisson formula.

This classical result will be utilized and generalized here.

We begin by supposing $\phi$ to be as in 7.4 , but we suppose $\phi$ vanishes on a 
neighborhood of 1 in $\Gamma$. Be it noted that we do not require $\phi$ to vanish on an open set of $\Delta$. For each $\rho$ in $\Delta, \phi$ vanishes on a set of positive $\mu_{\rho}$ measure, by 5.7. Hence the right side of $5.73(\zeta=\rho)$ is $-\infty$, and so (by 5.8) the left side also. Thus $\phi(\rho)=0$ for every $\rho$.

It follows that the holomorphic function 7.41 vanishes along the positive real axis, and hence $\phi\left(\rho^{0+i v}\right)=0$. By $7.2, \phi$ vanishes all along $\rho^{0} \Gamma^{\rho}$ for every $\rho(0 \leqq \rho \leqq 1$, in $\Delta)$.

If we define $\Gamma_{0}$ as the closed subgroup which is the limit of all these $\rho^{0} \Gamma^{\rho}$, 8.1

$$
\Gamma_{0}=\Gamma \cap\left(U \rho^{0} \Gamma^{\rho}\right)^{-}
$$

then $\phi$ vanishes on $\Gamma_{0}$. The reasoning can be repeated for every point in $V$, whence

8.11. Lemma. If $\phi=0$ on a neighborhood $V$ of 1 in $\Gamma$, then $\phi=0$ on $V \Gamma_{0}$.

The subgroup 8.1 may not be all of $\Gamma$. For example, let $G$ be the class of pairs of integers, with $G$ those pairs $(m, n)$ with $m>0$ or $m=0$ and $n>0$. Let $\alpha(m, n)=z^{m}, \beta(m, n)=w^{n}$ where $z, w$ are complex numbers of absolute value not greater than 1 . Then

$$
\phi(z, w)=z g(w)
$$$$
(|z|=|w|=1),
$$

where $g$ is any continuous function, is a representative function ( $\Gamma$ is the torus). If $g$ vanishes on an open set, so does $\phi$, but still $\phi$ need not vanish (identically). We leave it to the reader to find 8.1. $\phi=0$.

8.2. Theorem. If $\Gamma_{0}$ (see 8.1) $=\Gamma$ and $\phi$ vanishes on a nonvoid open set, then

This is obvious.

Proposition 8.2 says a lot more than the following, which is nevertheless of interest.

\subsection{Theorem. If $\Gamma_{0}=\Gamma$ then $A_{0}$ and $A_{1}$ are integral domains.}

Proof. If $\phi \psi=0$, and $\phi \neq 0$, then $\psi$ vanishes on an open subset of $\Gamma$ (indeed, even of $\Delta$ ). Then 8.2 can be applied.

What conditions imply $\Gamma=\Gamma_{0}$ ? We content ourselves with a sufficient condition.

8.4. Theorem. Suppose that for every $z$ in $G(z \neq 1)$ there is a real-valued, continuous homomorphism $h$ on $G$ such that $h(z) \neq 0$ and $h(x) \geqq 0$ for each $x$ in $G_{+}$. Then $\Gamma_{0}=\Gamma($ see 8.1$)$ so that 8.2, 8.3 hold.

Proof. For each such $h$ we can make a $\rho$ in $\Delta$ by defining $\rho(x)=e^{-h(x)}$. It follows that $z$ is excluded from $G^{\rho}$, whence the intersection of all these $G^{\rho}$ is the identity, in $G$. For such $\rho$ as these, the subset $\rho^{0} \Gamma^{\rho}$ of $\Delta$ coincides with the subgroup $\Gamma^{\rho}$ of the boundary $\Gamma$. The closure of the union of the $\Gamma^{\rho}$ is all 
of $\Gamma$. (This property is the dualized form of the stated property of the $G$.) Thus $\Gamma_{0}=\Gamma$.

The situation described in 8.4 can be created very easily. Let $T$ be any set, and let $G$ be any linear space of real-valued functions on $T$, and let $G_{+}$ be the class of non-negative functions. Define the discrete topology in $G$. Then the conditions 2.1-2.3 are fulfilled. Moreover, for $z \in G, z \neq 0$, there is a $t$ in $T$ such that $z(t) \neq 0$, while $x(t) \geqq 0$ if $x \geqq 0$. Thus 8.4 applies, and the ensuing ring $A_{0}$ is an integral domain.

8.5. Corollary. There exist Banach algebras $A_{0}$ of arbitrarily high dimension which are semi-simple operator algebras in a Hilbert space, and which are integral domains.

\section{BIBLIOGRAPHY}

I. Richard Arens and I. M. Singer, Function values as boundary integrals, Proc. Amer. Math. Soc. vol. 5 (1954) pp. 735-745.

II. Harald Cramér, Mathematical methods of statistics, Princeton, 1946.

III. L. H. Loomis, An introduction to abstract harmonic analysis, New York, 1953.

IV. Leon Pontrjagin, Topological groups, Princeton, 1939.

V. André Weil, L'intégration dans les groupes topologiques, Actualités Scientifiques et Industrielles, no. 869, Paris, 1940.

VI. Oscar Goldman, Ordered abelian groups, Bull. Amer. Math. Soc. Abstract 58-6-574.

VII. George Mackey, The Laplace transform for locally compact abelian groups, Proc. Nat. Acad. Sci. U.S.A. vol. 34 (1948) pp. 156-162.

University of CALIFornia, Los ANgeles, Calif. 